\title{
Lobectomy with pathological examination in lung cancer patients who recovered from COVID-19
}

\author{
Andrey Nefedov $^{1} \cdot$ Makhmud Mortada $^{1}$ (1) $\cdot$ Tatyana Novitskaya $^{1} \cdot$ Alexey Patsyuk $^{1} \cdot$ Andrey Kozak $^{1} \cdot$ Piotr Yablonskii $^{1}$
}

Received: 3 March 2021 / Accepted: 31 March 2021 / Published online: 9 April 2021

(c) The Japanese Association for Thoracic Surgery 2021

\begin{abstract}
We present two cases of lobectomy in lung cancer patients who recovered from COVID-19 before surgical treatment. In both cases, video-assisted thoracoscopic surgery was initiated and hilar fibrosis was detected; as a result, conversion was performed in one case. There were no postoperative complications and mortality. Also, we demonstrate the results of pathological examination in patients who have recovered from COVID-19.
\end{abstract}

\section{Introduction}

Up till now, there have been insufficient data on optimal treatment modality, time of surgery, preferred surgical accesses, risks of perioperative complications, and mortality for non-small cell lung cancer (NSLSC) patients who recovered from COVID-19 [1]. A recent report showed successful lobectomy by video-assisted thoracoscopic surgery (VATS) in a patient with lung cancer [2]. However, the technical difficulty in dissection of the hilum structures was documented. We report on a retrospective description of two cases of lobectomy with the presentation of the results of pathological examination in this category of patients.

\section{Case 1}

A 65-year-old nonsmoking woman suffered from fever and mild shortness of breath for 5 days. A nucleic acid test of COVID-19 was positive. Chest computed tomography (CT) showed an incidental finding of a $1.5 \mathrm{~cm}$ nodule in the right lower lobe (cT1bNOM0) and bilateral ground glass opacities (GGO) up to $15 \%$ of the lungs parenchyma (Fig. 1a). Within 10 days, she was treated for a moderate COVID19 at the hospital and was discharged home in satisfactory condition. Six weeks after her recovery, she was admitted

Makhmud Mortada

mortadamm92@gmail.com

1 St. Petersburg State Research Institute of Phthisiopulmonology, Ligovskiy ave, 2-4, St. Petersburg 191036, Russia for examination without clinical manifestations of COVID19, PCR test was negative, and forced expiratory volume in $1 \mathrm{~s}$ (FEV1) was 2,57L (114\%). On chest CT scan, the initial GGO lesions were in slighter density and smaller range (Fig. 1b). We performed uniportal VATS right lower lobectomy and systematic lymphadenectomy. The surgeon described the mottling of some parts of the lung with hemorrhage-like areas, injection of superficial pleural vessels, fibrosis of tissues around the vessels and bronchi, and difficulties in dissecting tissues with a harmonic scalpel due to their stiffness and density (Fig. 2). The operating time was $190 \mathrm{~min}$ due to severe fibrosis around hilar structures and blood loss was $50 \mathrm{ml}$. There were no postoperative complications, and the drain was removed on the 2 nd day. The patient was discharged on the 7 th postoperative day. Histologically, the tumor was lung adenocarcinoma pT1cN2 $(8 / 28)$ with metastases in paratracheal lymph-node group, and in the surrounding lung tissue, we revealed diffuse alveolar damage (DAD) (Fig. 3a), growth of granulation tissue in the lumen of the alveoli (Fig. 3b), and fibrosis foci in the lung tissue at the end of the proliferative phase (Fig. 3c).

\section{Case 2}

A 60-year-old male patient with a history including smoking for 10 years, peptic ulcer disease, and radiation therapy for prostate cancer. At follow-up PET/CT showed a peripheral tumor $3.5 \times 2.5 \mathrm{~cm}$ in right lower lobe. A month later, the patient consulted a doctor about symptoms of fever and dry cough. A nucleic acid test of COVID-19 was positive. CT showed minimal GGO up to $5 \%$ of the lungs volume. He 
Fig. 1 Lung CT scans. Initial images of peripheral mild GGO (a). CT scan the day before surgery with a pulmonary nodule and slighter GGO lesions (b)

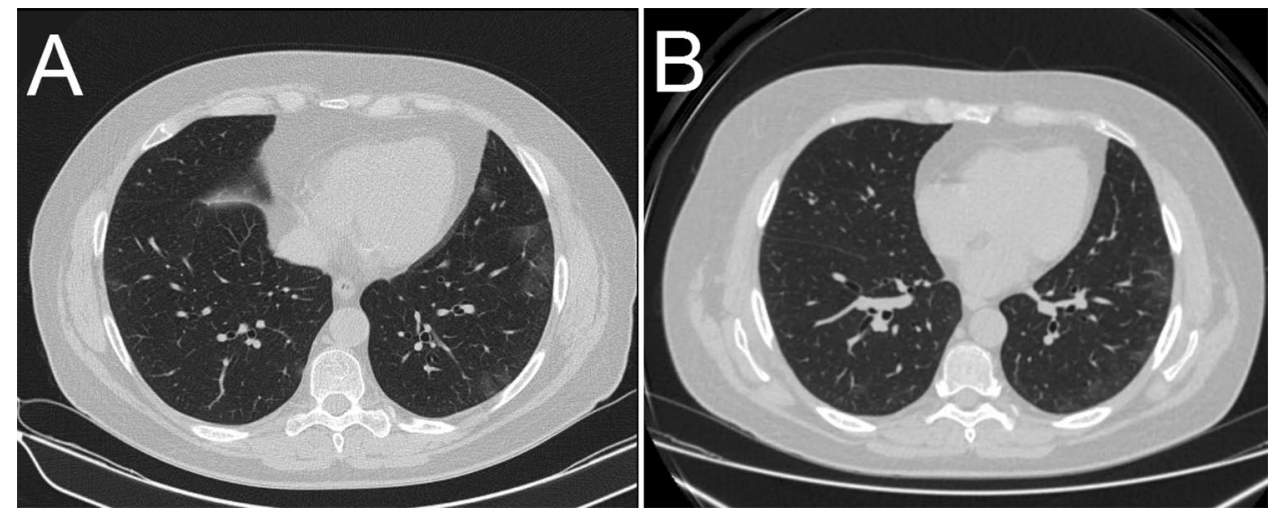

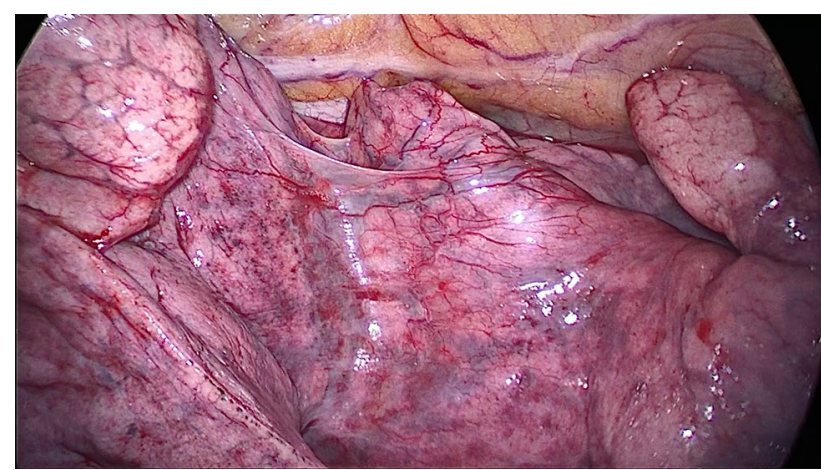

Fig. 2 Initial view of right lung has a mottled appearance with hemorrhage-like areas and injection of superficial pleural vessels

received outpatient treatment for COVID-19. Control chest CT showed no new changes; FEV1 $=2,28 \mathrm{~L}$ (102\%); transbronchial biopsy verified NSCLC. Six weeks after recovery, lobectomy with systematic lymphadenectomy began as uniportal VATS and was converted to thoracotomy due to dense hilar fibrosis and the impossibility to safely expose the pulmonary artery. There were no adhesions and effusion in the pleural cavity and no lymph node was enlarged. The operative time and blood loss were $270 \mathrm{~min}$ and $150 \mathrm{ml}$, respectively. The postoperative period passed without complications, and the drainage was removed on the 3rd day. The patient was discharged on the 8th day after surgery. Histologically, the tumor was primary squamous cell carcinoma pT1cN0 (0/21), and the surrounding lung parenchyma was at the proliferative phase of DAD (Fig. 3d).

\section{Discussion}

The widespread use of CT to detect COVID-19 pneumonia can lead to incidental detection of lung cancer, as illustrated in the first case. Due to the limited data, the thoracic guidelines recommend weighing the risk-benefit ratio to make decisions [1]. The optimal period of surgical treatment after recovery from COVID-19 is not known; in our cases, it comprised 6 weeks after recovery. By this time, radiological changes in the lungs had significantly regressed, which was shown in studies for patients with mild form [3]. Despite this, pathological changes were still revealed histologically in the form of a proliferative phase of diffuse alveolar damage with the identification of foci of fibrosis. Polak in his review showed similar data that after about 3 weeks, some patients (22\%) develop fibrotic changes in the lung tissue [4]. This is likely to be the reason of difficulty during dissection of the hilum structures, which caused the prolongation of the operation time, and the need for conversion to thoracotomy in one case. Despite the fact that the development of COVID-19 in the perioperative period showed extremely high morbidity and mortality, these results were not observed in our and in previous case reports in patients who had suffered from COVID-19 before the surgery $[2,5]$. These data demonstrate the feasibility of lobectomy with systematic lymphadenectomy in patients with lung cancer who have recovered from COVID- 19 . 


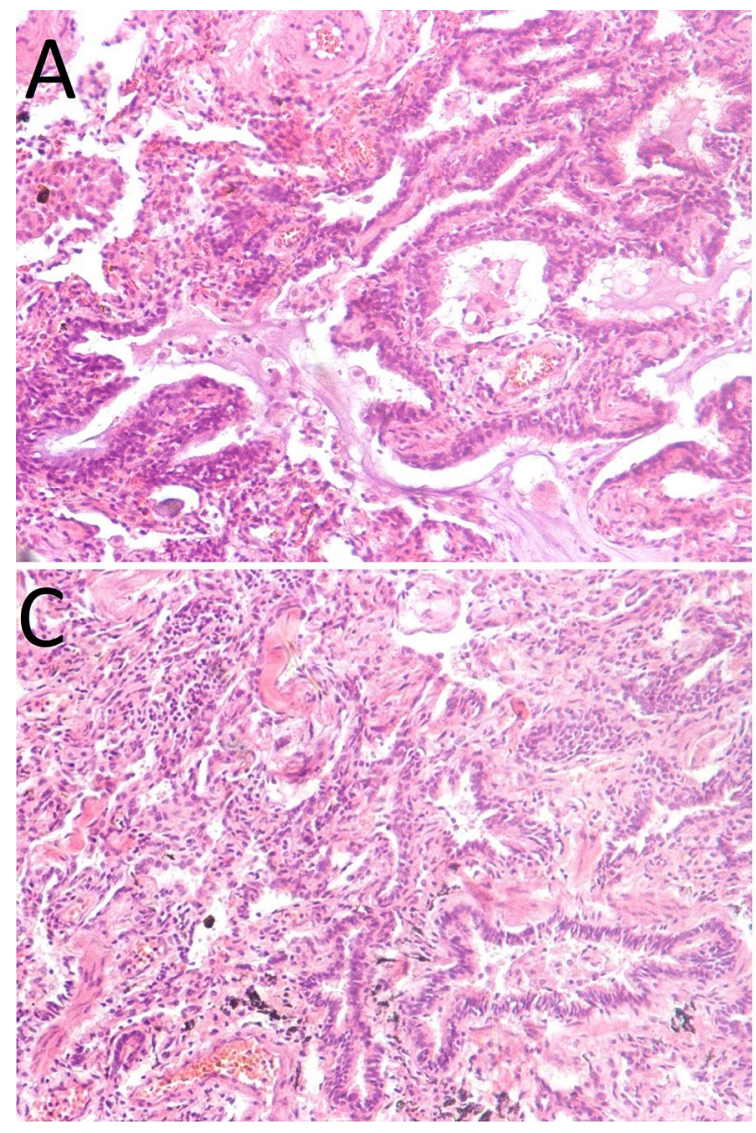

Fig. 3 Histologic changes the surrounding lung parenchyma, $\times 100$, hematoxylin, and eosin. DAD, mild fibrosis of the interalveolar septa, mild inflammatory infiltration; the lumens of the bronchioles are dilated, contain mucus and a small number of macrophages; microangiopathy, diapedetic hemorrhages around the alveoli (a). Growth of granulation tissue in the lumen of the alveoli, the abundance of con-

\section{References}

1. British Thoracic Society. Lung cancer and mesothelioma service guidance during the COVID-19 pandemic. https://www.britthoracic.org.uk/about-us/covid-19-information-for-the-respi ratory-community. accessed 15 Jul 2020.

2. Testori A, Perroni G, Voulaz E, et al. Pulmonary lobectomy after COVID-19. Ann Thorac Surg. 2020;S0003-4975(20):31545-9.

3. Liu C, Ye L, Xia R, et al. Chest computed tomography and clinical follow-up of discharged patients with COVID-19 in Wenzhou City, Zhejiang. China Ann Am Thorac Soc. 2020;17(10):1231-7. https://doi.org/10.1513/AnnalsATS. 202004-324OC.

4. Polak SB, Van Gool IC, Cohen D, et al. A systematic review of pathological findings in COVID-19: a pathophysiological

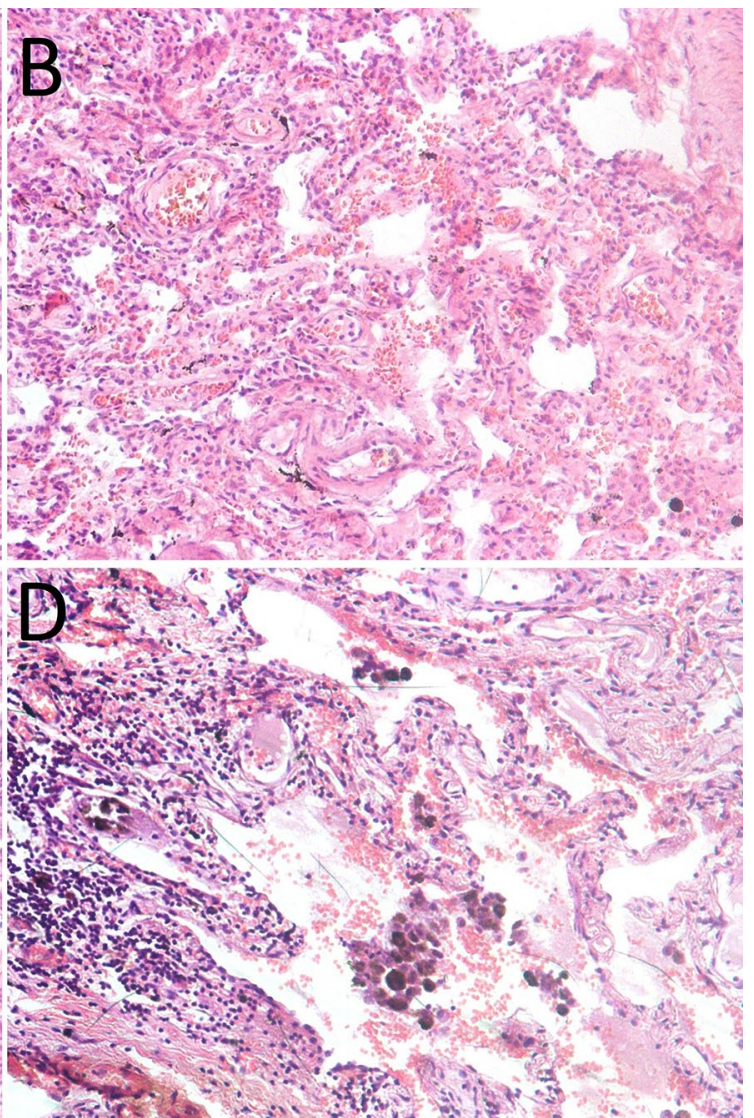

gested vessels, and diapedetic hemorrhages (b). Fibrosis foci in the lung tissue at the end of the proliferative phase (c). DAD, proliferative phase: focal intraalveolar edema, focal accumulations of alveolocytes, macrophages, erythrocytes, fibrin in the alveoli; focal perivascular accumulations of lymphocytes (d)

timeline and possible mechanisms of disease progression. Mod Pathol. 2020;33(11):2128-38. https://doi.org/10.1038/ s41379-020-0603-3.

5. Cai Y, Hao Z, Gao Y, et al. Coronavirus disease 2019 in the perioperative period of lung resection: a brief report from a single thoracic surgery department in Wuhan, People's Republic of China. J Thorac Oncol. 2020;15(6):1065-72. https://doi.org/ $10.1016 /$ j.jtho.2020.04.00.

Publisher's Note Springer Nature remains neutral with regard to jurisdictional claims in published maps and institutional affiliations. 